\title{
Brief Report: Renal Failure on Melanoma an Experimental Overview of Melanin and Immuno- Complexes Deposition
}

\author{
Bruno Campos Fontoura ${ }^{1}$, Bruno Ribeiro Cruz², Leandro Cavalcante Lipinski ${ }^{3}$, Ângelo César D’Urso \\ Panerari $^{4}$, Daniel Fernandes ${ }^{5}$ and Giovani Marino Favero ${ }^{1 *}$ \\ ${ }^{1}$ Pathophysiology of the immune response Laboratory, Brazil \\ ${ }^{2}$ Hematology Laboratory, Biological and Health Sciences Division, Brazil
}

${ }^{3}$ Surgical Technique Laboratory, Brazil

${ }^{4}$ Departament of Medicine, UNICESUMAR, Faculdades Ingá, Brazil

${ }^{5}$ Department of Pharmacology, Brazil

*Corresponding author: Giovani Marino Favero, Department of General Biology, Parana, Brazil

\section{ARTICLE INFO}

Received: 幽 July 10, 2019

Published:

Citation: Bruno Campos F, Bruno Ribeiro C, Leandro Cavalcante L, Ângelo César D’Urso P, Daniel F, Giovani Marino F. Brief Report: Renal Failure on Melanoma an Experimental Overview of Melanin and Immuno-Complexes Deposition. Biomed J Sci \& Tech Res 19(4)-2019. BJSTR. MS.ID.003345.
ABSTRACT

Background: Metastatic melanoma progression induces renal insufficiency, with a sequence of infections due to immunological involvement, ascites, hypertension and subsequent hypotension associated with sepsis. The aim of this study was to evaluate biochemical renal damage markers in an experimental model of melanoma in mice.

Methods: Animals were divided into three groups: control, B16F10 (melanoma) cell implantation and animals receiving daily injections of melanoma culture supernatant. It was evaluated in two periods ten and twenty days. Renal function were evaluated by urinary markers. Interleukin-18 were evaluated by ELISA.

Results: There was loss of renal function in mice with melanoma evaluated by urinary markers; the daily application of melanin and other factors derived from the melanoma supernatant did not alter the renal function. Melanoma bearing mice, after 20 days, presented a significantly value of Interleukin-18.

Conclusion: Renal function is gradually changed in melanoma and is probably associated with immune complexes than circulating melanin.

\section{Background}

Melanoma is the skin cancer with highest mortality potential, representing about $4 \%$ of all skin tumors. This tumor is among the causes of Membranous Nephropathy. This is a disease characterized by diffuse thickening of the glomerular capillary wall due to the accumulation of electrodens immunglobulin depositis along the subepithelial side of the basement membrane. The glomerular wall becomes defective, which in the long term may lead to loss of renal function [1]. In addition to the deposition of immunoglobulin complexes, the abundant secretion of melanin by this type of tumor leads to renal failure. Notably, the clinical progression of patients with metastatic melanoma are renal insufficiency, with a sequence of infections due to immunological involvement, ascites, hypertension and subsequent hypotension associated with sepsis. Melanoma, despite having characteristics of differentiated melanocyte as the secretion of melanin, is a tumor with a high degree of aplasia. The endocrine action and abundant secretion of melanin promotes a break in homeostasis and the kidneys are commonly affected first. In a case report, clinicians described a patient who presented Renal Failure due to deposition of immuno-complexes without apparent cause, after six months the diagnosis of metastatic melanoma was described, the first manifestation being nephropathy [2]. In patients and melanoma-bearing mice experimental models the observation of melanin deposits is common over the abdominal cavity. 
Despite the practical-clinical knowledge that Melanoma is able to secrete abundantly melanin and to promote relative toxicity, there are a few descriptions of Melanuria in this type of patient, as a clinical report published in 2008, where it were observed diffuse tubular injury, accumulation of melanin in tubular epithelial cells, diffuse melanose in a case of generalized metastases [3]. Melanin is a protein with relatively high weight and its presence in the circulation is extremely rare, described so far, in patients with diffuse melanose. The size of the protein and its chemical characteristics have nephrotoxic characteristics. Currently, due to little discussion on the relevance of melanin deposits, the presence of this compound in the urine is only reported when the color change in this excretion. One of the new markers for Renal Failure and Acute Glomerular Necrosis is the increased concentration of circulating IL-18, which is also present in most tumors, as it is a proinflammatory cytokine. [4] Few studies have shown experimentally and/or chronologically the effects of melanoma on renal function. Thus, we aimed the evaluation of nephrotoxic events in a classic model of melanoma in mice.

\section{Material and Methods}

Ethical precepts in the use of animals and had its research project approved by the Animal Studies and Research Committee, under the CEUA/UEPG Process No. 048/2017.

\section{Cell Culture}

The B16F10 melanoma cells line, were kindly donated by the researcher of the Cancer Institute of the State of São Paulo (ICESP) Dr. Andréia Hanada Otake. The culture conditions were: RPMI 1680 medium supplemented with $10 \%$ Fetal Bovine Serum. Cells were kept in oven with $5 \% \mathrm{CO}_{2}$ atmosphere at $37^{\circ} \mathrm{C}$ in $125 \mathrm{~cm}^{2}$ culture flasks.

\section{Determination of Melanin Estimation in B16F10 Cell Supernatant}

B6F10 cells naturally secrete melanin, with a poorly defined cyclic variation. Only as an estimative control of melanin was the evaluation of turbidimetry associated to the cellular amount, so that there was a pattern of injections in the mice. Briefly, B16F10 cells (1x106 / flask) were seeded and incubated for 48h. At the end of this period, the supernatant was removed and filtered for the cleavage of possible cell or cell debris present in the medium. The relative cell count was then performed. The optical density of the supernatant was then measured at $490 \mathrm{~nm}$. This step was performed in the indirect quantification of melanin and maintenance of the application pattern in the animals of this experimental group.

\section{Animal Model}

Sixty mice (mus musculus) were used from the UEPG Vivarium without genetic alteration. The line used was C57BL/6, with 60 days of age and approximate weight of $22 \mathrm{~g}$. Feeding and water support ad libitum.

\section{Tumor Implantation}

B16F10 cells (murine melanoma) were cultured to confluence. For cell implantation, the C57BL / 6 mice had the posterior flank region tricostomized. B16F10 cells (5x104) were injected into the right posterior part of the mice. This type of tumor has accelerated growth, being visible in less than 10 days after the implant. The animals were divided into three experimental groups: 1 . Control group - naive; 2 . Group of animals without tumor, but with daily injections of melanoma-containing melanoma culture supernatant; 3. Group of animals with tumor, 5x104 implant of B16F10 cells.

\section{Renal Function Evaluation}

Two periods were evaluated after 10 days and 20 days of tumor implantation or treatment with the melanoma culture supernatant. At the end of these periods a puncture was performed for blood collection and cardiac puncture of the animals. Blood was centrifuged and routine procedures were performed for the quantification of Serum Creatinine, Blood Urea Nitrogen and Interleukin-18 (Mouse IL-18 Platinum ELISA BMS618 / 3 / BMS618 / 3TEN from eBioscience kit). Statistical Analysis For the cell assays with the different treatments the ANOVA test will be used. Both statistical analyzes will be developed in the GraphPad software (Prism 4.0), values of $p<0.05$ will be considered significant.

\section{Results}

After tumor implantation and daily treatment with melanoma culture supernatant, we had two experimental evaluation periods, after 10 and 20 days. (Figure 1) shows the results found in the serum evaluation of Urea and Creatinine. Taking into account the reference value as the control group and a comparative evaluation between the two experimental groups, we can verify that there was statistical difference between the Melanoma and supernatant groups after 10 days of the experiment. The second period evaluated was after twenty days of experimentation, it can be noticed a greater presence of these markers in the animals with melanoma. Recently, Interleukin-18 has been used as a possible marker of acute renal failure. Figure 2 shows the IL-18 values of the mice after 20 days of experiment. The animals that had the Melanoma after 20 days presented a significantly higher value of this substance in relation to the other groups. 

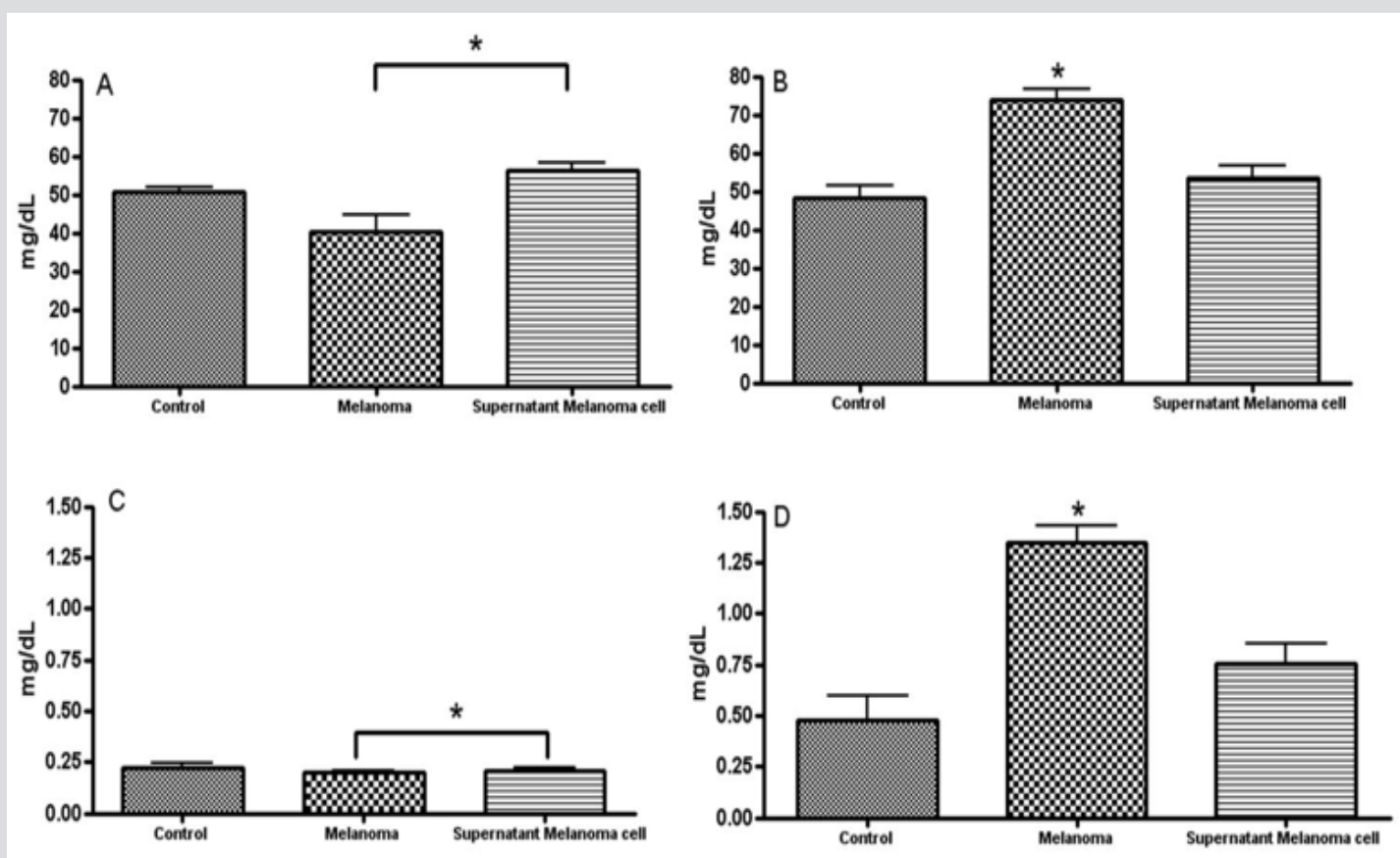

Figure 1: Biochemical evaluation of plasmatic Urea.

A. Creatinine

B. from C57BL/6 mice undergoing B16F10 melanoma implantation or daily doses of B16F10 melanoma culture supernatant (XX uL, s.c. or ip?) after 10 days (A and C) and 20 days (B and D) of experimentation. Recomendo citar aqui o teste estatístico usado * $\mathrm{p}<0.05$

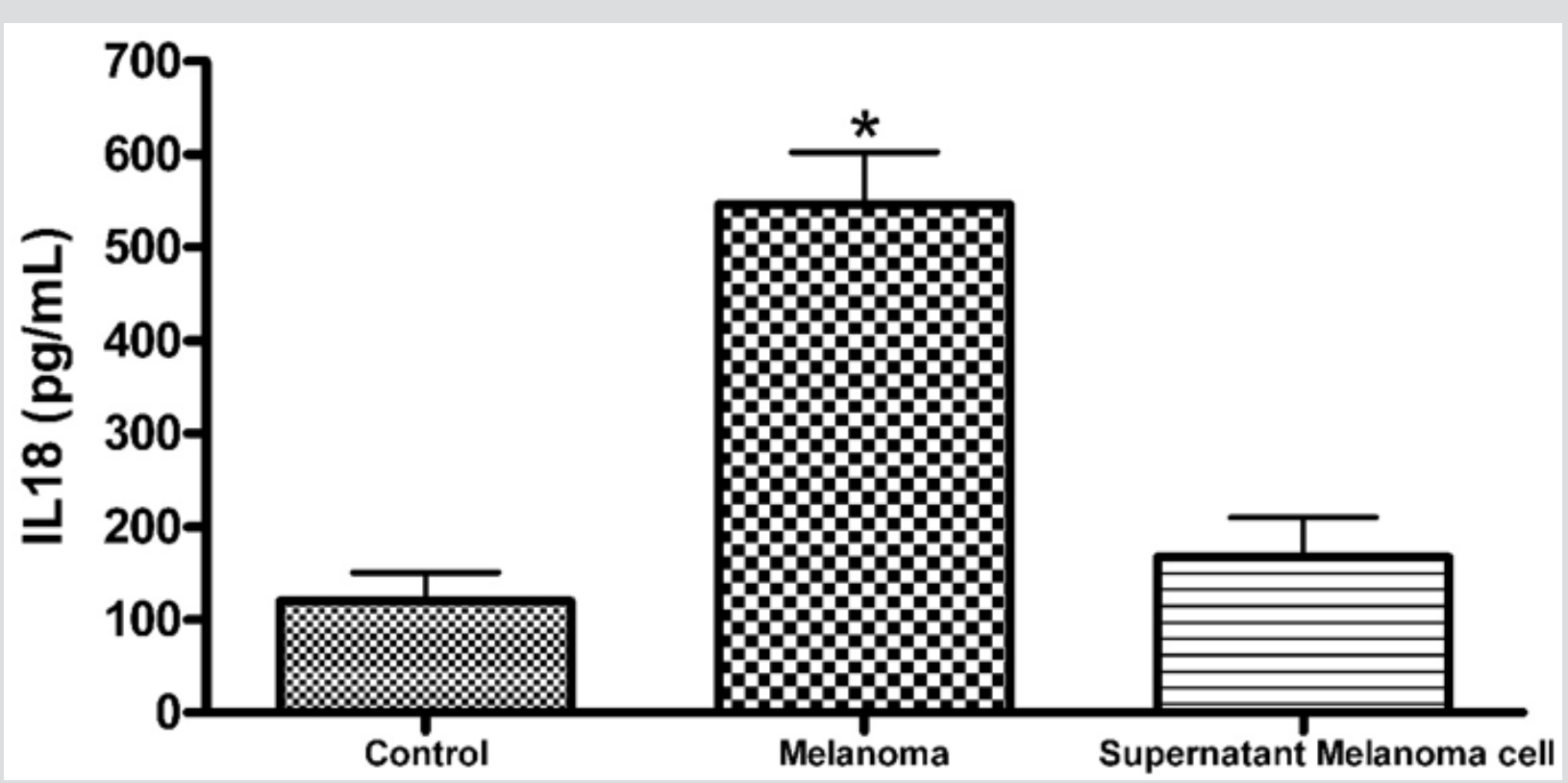

Figure 2: Interleukin-18 Serum quantification in untreated, melanoma-bearing mice (20 days after injection of 5x104 B16F10 cells) and treated with B16F10 culture supernatant for twenty days. Recomendo citar aqui o teste estatístico usado * $\mathrm{p}<0.05$.

\section{Discussion and Conclusion}

The significant increase in circulating IL-18 in animals with melanoma after 20 days of the injection of the B16F10 melanoma cells. The complexity and interaction of the neoplastic mass was superior, in effect, when compared to the daily treatment of the melanoma culture (B16F10) supernatant, which leads to the hypothesis that the cells when incorporated into the organism have a greater systemic activity. Serum creatinine is an important marker of glomerular filtration rate. In our experimentation we observed that after ten days of the beginning of the tests, there was 
a significant difference between the melanoma and supernatant groups, being the group without tumor with a higher value of this marker. This difference may be related to the amount of cellular metabolites in the medium and acid $\mathrm{pH}$, which is capable of increasing renal activity [5]. The same evaluation was performed after twenty days. At this period, the animals affected by the tumor had an increased serum creatinine value in relation to the other groups. The most likely explanation is that, after twenty days the tumor is already well established, with a very apparent size, with its tissue distributed with that of the host, so the secretion enlargement metabolites, $\mathrm{pH}$ acidification and other associated biochemical events production and secretion of this marker. In parallel with the evaluation of serum creatinine, circulating urea, , was also evaluated.

In the first evaluated time period the treatment with the melanoma culture supernatant increases as the creatinine level in relation to the group carrying melanoma. The increase may be justified by the daily injections of supernatant, into the peritoneum. The supernatant is abundant in proteins which are captured by the lymphatic circulation and later filtered in the kidneys. After the twenty days and with the clear and visual tumor progression, the urea values were higher in this experimental group. High values of urea are related to infections, liver changes, excess protein in the diet and, in this case, cancer [6]. Follow-up melanoma patients and

\section{ISSN: 2574-1241}

DOI: 10.26717/BJSTR.2019.19.003345

Giovani Marino Favero. Biomed J Sci \& Tech Res CC) This work is licensed under Creative

Submission Link: https://biomedres.us/submit-manuscript.php other types of tumors usually focuses specifically on the neoplastic mass. This study addressed the importance of systemic assessment and a differentiated view of the kidneys on melanoma bearing patients. With the advancement of biochemical quantification technologies and organ-to-organ therapy, it is believed that differentiated care with different types of physicians can improve and extend the survival of these patients.

\section{References}

1. Pozdzik A, Brochériou I, David C, Touzani F, Goujon JM, et al. (2018) Membranous Nephropathy and Anti-Podocytes Antibodies: Implications for the Diagnostic Workup and Disease Management. Biomed Res Int. (2018) 6281054 .

2. Faria TV, Baptista MA, Burdmann EA, Cury P (2010) Glomerular deposition of immune complexes as a first manifestation of malignant melanoma a case report. Renal Failure 32(10): 1223-1225.

3. GAMBICHLER T, STIICKER M, Kerner K, Weiner S, Waldherr R, et al. (2008) Acute Kidney Injury in a Patient with Melanuria, Diffuse Melanosis, and Metastatic Malignant Melanoma. Am J Clin Dermatol 9(4): 267-270.

4. Hutton Hl, Alikhan Ma, Kitching Ar (2018) Inflammasomes In the Kidney. Expsuppl 108: 177-210.

5. Huber K, Breves G (1999) Influence of dietary phosphorus depletionon central pathways of intermediary metabolism in rats. ArchTierernahr 52(4): 299-309.

6. Levey AS (1990) Measurement of renal function in chronic renal disease. Kidney Int 38(1): 167-184.

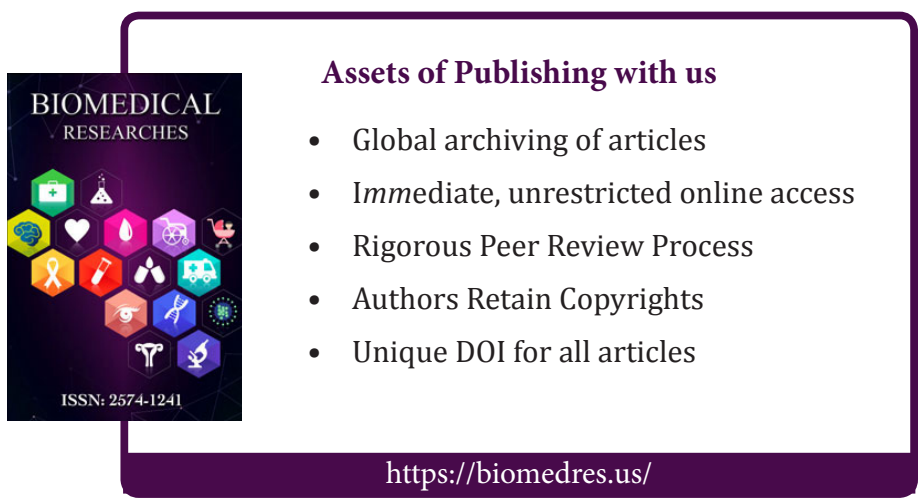

